\title{
Demokratieprobleme des Steuerstaates im 21. Jahrhundert: Eine Einführung
}

\author{
Sebastian Huhnholz • Daniel Mertens • Thomas Rixen
}

Online publiziert: 22. Februar 2018

(C) Deutsche Vereinigung für Politikwissenschaft 2018

Wir können gegenwärtig eine Neujustierung des Verhältnisses von Demokratie, Staatsfinanzierung und internationaler Politik beobachten. Insbesondere die Doppelkrise von Demokratie und Kapitalismus, wie sie auch in dieser Zeitschrift verhandelt worden ist (Biebricher und Vogelmann 2014; Rittberger und Schimmelfennig 2015), hat einen Kristallisationspunkt in den öffentlichen Finanzen gefunden, der sich in zahlreichen gesellschaftlichen und politischen Kontroversen und Konflikten widerspiegelt. Während in Deutschland die „schwarze Null“ (Haffert 2016) zur festen Zielgröße geworden ist, vor deren Hintergrund im Wahlkampfjahr über die Verwendung ,sprudelnder Steuereinnahmen“ gestritten wurde, könnte die Diskrepanz zu vielen Ländern Europas eklatanter kaum sein. In unheiliger Allianz mit der Flüchtlingspolitik hat die Krise der Staatsfinanzierung als Krise des weiteren Finanzsystems zu schwerwiegenden Turbulenzen in den europäischen Parteiensystemen und den sie tragenden Demokratien geführt. Und wer die internationale Dimension der aktuellen Herausforderungen für den Nexus von Staatsfinanzierung und Demokratie in den kapitalistisch organisierten Gesellschaften zu vergessen droht, wird durch regelmäßige Skandale wie die Lux Leaks, Panama Papers oder Paradise Papers daran erinnert.

Diese zum Teil neuen, teilweise aber auch durch die andauernde Krise nur zugespitzten Entwicklungen stoßen in der Öffentlichkeit auf großes Interesse und werden populärwissenschaftlich breit rezipiert. Seltener finden sie sich allerdings in der

\footnotetext{
S. Huhnholz

Hannover, Deutschland

D. Mertens $(\square)$

Frankfurt, Deutschland

E-Mail: mertens@soz.uni-frankfurt.de

T. Rixen

Bamberg, Deutschland
} 
fachinternen, politikwissenschaftlichen Debatte wieder - ein Desiderat, das dieses Schwerpunktheft mit einem Konzept aufgreift, das trotz seiner demokratietheoretischen Relevanz in der Disziplin kaum Gehör findet: dem des Steuerstaates.

\section{Der Steuerstaat ...}

Steuerpolitik organisiert heute nicht nur ,die Sekundärverteilung in der Gesellschaft“ (Bieling 2015, S. 5), sondern der „Steuerstaat als Staatsform“ (Isensee 1977) wirkt auch auf alle Einzelheiten der ,primären “ Verteilung, d.h. der markt- und vermögensvermittelten Verhältnisse, ein. Der Steuerstaat ist der funktionale Begleiter des kapitalistischen Wirtschaftsmodells (Weber 2014 [1919/20]; Schumpeter 1918). Die Steuer als voraussetzungslose, d.h. nicht an konkrete Gegenleistungen gebundene Zwangsabgabe ermöglicht den umfassenden Schutz des Privateigentums (statt vieler: Preuß 1981) und ist politisch auf den ökonomisch erfolgreichen Einsatz des privaten Kapitals angewiesen (Huhnholz 2017). Steuerstaat und Wirtschaftsbürger bilden eine fundamental auf Wirtschaftswachstum konditionierte „Erwerbsgemeinschaft““ (Kirchhof 1996, S. 33).

Neben diese politökonomische Dimension tritt auch eine demokratietheoretische. Als „Staatsspiegel“ (Reimer 2013, S. 125) lässt jede Fiskalverfassung Rückschlüsse auf Umfang und Zustand politischer Rechte und bürgerliche Freiheiten zu (Gould und Baker 2002; Mann 1978; Priddat 2009, S. 31-32). Insbesondere die Steuer gilt für verschiedenste Theorietraditionen als geradezu perfektes ökonomisches Partizipationsmedium der repräsentativen Demokratie (z. B. Hettich und Winer 1999; Levi 1988; Ross 2004). Die markante Revolutionslosung No taxation without representation! kommt bis weit in unsere Zeit hinein einem demokratischen Glaubensbekenntnis gleich (Huhnholz 2015).

Der Steuerstaat ist somit derjenige Staat, der sich durch die hoheitliche Auferlegung von Geldleistungspflichten auf privatwirtschaftliche Gewinne und deren Verwendung finanziert (vgl. Schumpeter 1918; Grauhan und Hickel 1978). Gleichwohl lässt der vorliegende Schwerpunkt eine breitere Definition der Steuerstaatlichkeit $\mathrm{zu}$, um einerseits das erweiterte - und letztlich steuerstaatlich rückversicherte - fiskale Budget wie Sozialversicherungsbeiträge und Kredite zu berücksichtigen und andererseits die Ausgaben des Staates in den Blick zu nehmen. ${ }^{1}$ Damit berücksichtigen wir nicht nur die gängige Forschungspraxis, die moderne Wohlfahrtsstaaten nahezu intuitiv mit Steuerstaaten gleichsetzt (Wagschal 2003; Steinmo 1993), sondern kommen auch Kritiken an einer Überhöhung der Steuerstaatsnorm entgegen (Gawel 2000; Knill 2013; Sacksofsky und Wieland 2000), die noch immer in einem „,innigen wechselseitigen Funktionalzusammenhang“ (so bereits Goldscheid 1976 [1926], S. 254-255) mit den im Folgenden herausgestellten Demokratieproblemen gesehen werden müssen.

\footnotetext{
1 Die Finanzwissenschaft bezeichnet als Steuer im engen Sinne lediglich die oben bereits erwähnte geldliche Zwangsabgabe ohne konkreten Gegenleistungsanspruch und unterscheidet diese von Beiträgen (bei denen es eine gruppenäquivalente Gegenleistung gibt, Beispiel Sozialversicherung) und Gebühren (direkte individuelle Gegenleistung, Beispiel Müllabfuhrgebühren) (Homburg 2000).
} 


\section{2 ... und seine alten und neuen Demokratieprobleme}

Die Problematisierung des Verhältnisses von Demokratie und Steuerstaat zieht in der Regel zwei Wirkungsweisen in Betracht. Entweder die Frage, welche Probleme der kapitalistisch fundierte Steuerstaat für das Funktionieren der Demokratie verursacht, oder, umgekehrt, wie die Demokratie den Steuerstaat gefährden könnte.

In der politikwissenschaftlichen und politökonomischen Literatur wurde bis in die achtziger und neunziger Jahre des vorigen Jahrhunderts von der public choice-Schule vor allem die zweite Perspektive stark gemacht. Ihr Ausgangspunkt ist die Tatsache, dass bei öffentlichen Gütern eine individuelle Zurechnung von Kosten und Nutzen für die Bürgerinnen und Bürger nicht möglich (und in vielen Fällen auch nicht wünschenswert) ist. ${ }^{2}$ Bei Downs (1960) führt dies dazu, dass es unter der Annahme stimmenmaximierender Parteien und ,rational ignoranter" Wählerinnen und Wähler zu einem Gleichgewicht im politischen Wettbewerb kommt, in dem der öffentliche Haushalt systematisch zu klein (gemessen an den Präferenzen der Bürgerinnen und Bürger) ist. Demgegenüber sieht der deutlich größere Teil der public choice-Literatur unter der Annahme budgetmaximierender Politiker und Bürokraten, die unter dem Einfluss partikularer Interessen (rent-seeking) und umverteilungsfreundlicher (einfacher) Mehrheiten stehen, die Gefahr, dass der Steuerstaat zu groß wird und in die Verschuldung ausweicht (Buchanan und Tullock 1962; Brennan und Buchanan 1980 - dazu Huhnholz 2018a in diesem Heft).

Die neo-marxistische Literatur der 1970er-Jahre vertritt ebenfalls die These, dass die demokratisch vermittelten, sozialpolitischen Ansprüche der Bürger die öffentlich abschöpfbare Finanzkraft der kapitalistischen Wirtschaft übersteigen. Allerdings sieht sie darin, anders als die public choice-Tradition, keinen Defekt der Demokratie, sondern „Strukturprobleme des kapitalistischen Staates“ (Offe 1972), der tief in Marktprozesse eingreifen muss, um deren Funktionieren zu sichern. Dies wiederum verursache „Legitimationsprobleme im Spätkapitalismus“ (Habermas 1973 - dazu Buggeln 2018 in diesem Heft).

Seit den 1990er-Jahren und verstärkt seit den 2000er-Jahren treten neben diese älteren Debatten neue Demokratieprobleme des Steuerstaates, in denen die erste Perspektive - Gefährdung der Demokratie durch den Kapitalismus - eingenommen wird. Drei (miteinander verbundene) Themenkomplexe sind hier einschlägig.

Erstens dreht sich ein großer Literaturstrang um die Folgen der Globalisierung für die politische Handlungsfähigkeit von Nationalstaaten. Die Befürchtung ist, dass die ,entgrenzte“ Wirtschaft nicht mehr durch eine nationalstaatlich , begrenzte' Politik reguliert werden kann, oder gar unter die Aufsicht transnationaler Finanzakteure ohne demokratische Legitimation oder Kontrolle gestellt wird (z. B. Strange 1996; Genschel und Uhl 2006; zuletzt z.B. Offe 2016). Die Steuerstaaten seien angesichts international mobilen Kapitals in einen schädlichen Standortwettbewerb geraten (z.B. Rixen 2011 - dazu auch Rixen 2018 sowie Wollner 2018 in diesem Heft). Umverteilung und andere kollektive, aber nicht marktlich zu befriedigende

\footnotetext{
2 Genau mit dieser Eigenschaft öffentlicher Güter lässt sich deshalb die oben bereits angesprochene Voraussetzungslosigkeit der Steuer, also die Abwesenheit einer konkreten, individuellen Gegenleistung, wie sie in die rechtliche Definition eingeflossen ist, begründen.
} 
Interessen müssten um den Preis des wirtschaftlichen Niedergangs der Standortwettbewerbslogik untergeordnet werden, worin ein Verlust des Gehaltes nationalstaatlich verfasster Demokratie zu sehen sei (z. B. Streeck 1998). Demgegenüber wurde von anderen Autoren betont, dass die national verfasste Demokratie den Marktkräften der Globalisierung durchaus wirksam standhalten könne und das gefürchtete ,race to the bottom' sowie der damit einhergehende Kontrollverlust ausbliebe (für einen Überblick, siehe Genschel 2003).

Andererseits kann der wirtschaftlichen auch eine politische bzw. institutionelle Globalisierung bzw. Internationalisierung an die Seite gestellt werden. Im Zuge internationaler Kooperation und Institutionenbildung könnte ein ,Regieren jenseits des Nationalstaates“ (Zürn 1998) möglich werden, das die auf nationalstaatlicher Ebene verlorene Handlungsfähigkeit auf internationaler Ebene zurückholt, und so im besten Falle den demokratischen Präferenzen der Bürgerinnen und Bürger zur Durchsetzung verhilft. Insbesondere die Europäische Union biete sich hierfür in besonderer Weise an (z.B. Habermas 1998, 2013). Allerdings sind gegen diese Vorstellung skeptische Stimmen laut geworden, die zum einen darauf hinweisen, dass gerade in der EU, aber auch in anderen durchschlagskräftigen Institutionen, eine Agenda der negativen Integration zu Lasten der positiven vorherrsche und mithin die soziale Demokratie zu Lasten der liberalen Rechtsstaatlichkeit geschwächt werde (Scharpf 1999; Höpner und Schäfer 2012). Des Weiteren stellten sich angesichts des notwendigen Regierens auf mehreren Ebenen häufig Politikverflechtungsfallen und Demokratiedefizite ein (dazu Mertens 2018 in diesem Heft).

Zweitens ergeben sich aus einer in den letzten beiden Jahrzehnten in fast allen Industrieländern angewachsenen ökonomischen Ungleichheit Demokratieprobleme. Es ist gezeigt worden, dass es reicheren Menschen besser als ärmeren gelingt, ihre politischen Präferenzen in der Politik durchzusetzen (Gilens 2012) und dass die Wahl- und politische Beteiligung mit wachsender Ungleichheit abnimmt, wobei sich vor allem die einkommensschwachen Gruppen aus der politischen Beteiligung zurückziehen (Schäfer 2013). Der Steuerstaat ist in diese Entwicklungen besonders verwickelt. Einerseits ist der Anstieg der Ungleichheit durch die in den letzten drei Dekaden verfolgten Politiken der Liberalisierung, des Umbaus des Sozialstaates und der Steuersenkung mitverursacht worden (Piketty 2014; siehe auch Rixen 2018 in diesem Heft). Einige Beobachter haben bereits die Befürchtung geäußert, dass man es hier mit einem sich selbst verstärkenden Zirkel aus regressiveren Sozialund Steuerpolitiken, wachsender Ungleichheit sowie sinkender und verzerrter demokratischer Teilhabe zu tun habe (Stiglitz 2008). Andererseits gilt die Steuer- und Finanzpolitik von Steuerstaaten weiterhin als zentrales, wenn nicht als einziges Element in einer politischen Strategie gegen die wachsende Ungleichheit (Piketty 2014; Atkinson 2015), was möglicherweise aber nicht nur für Demokratien, sondern auch für Autokratien gilt (dazu Seelkopf 2018 in diesem Heft).

Drittens und letztens hat die globale und europäische Finanzkrise die Thematik der Instabilität des Kapitalismus erneut auf die Tagesordnung gebracht. Zwar diagnostizierte man zu Beginn der Krise die Rückkehr des zuvor verabschiedeten starken, d.h. handlungsmächtigen und interventionswilligen Staates (pars pro toto Hassel und Lütz 2010). Aber gerade in fiskalischer Hinsicht blieb immer weniger verborgen, dass der Staat, der derzeit wiederkommt, nicht derselbe ist, den man für 
verschwunden oder gar überwunden hielt (Buggeln 2012; Genschel und Schwarz 2013; Streeck und Mertens 2010). Neben die direkten finanzpolitischen Effekte der Krise trat die Inanspruchnahme der Staatshaushalte durch die Rettung, systemrelevanter "Finanzinstitutionen und in Folge dessen der europäisch-koordinierte Umbau des institutionellen Handlungsrahmens des Steuerstaates. Die daraus resultierenden Krisendiagnosen sind kontrovers verhandelt worden (Biebricher und Vogelmann 2014; Enderlein 2013; Streeck 2013), aber klar scheint, dass die Prozesse der ,Verrechtlichung ' und ,Technokratisierung ' vor allem der Einschränkung des fiskalischvermittelten demokratischen Handlungsspielraums Vorschub geleistet haben (zum Begriff der „fiskalischen Demokratie“ siehe Genschel und Schwarz 2013; Streeck und Mertens 2013; sowie Rixen 2018; Mertens 2018 und Huhnholz 2018a je in diesem Heft).

Die hier dargelegten Trends geben Anlass genug, den Steuerstaat wieder ins Zentrum politikwissenschaftlicher Analyse zu rücken. Zwar sah man den Steuerstaat seit jeher in der Krise (Drüen 2015; Grauhan und Hickel 1978; Herbert und Stiefel 1997; Huhnholz 2018b; Schumpeter 1918), weil sowohl die normative Voraussetzungslosigkeit der Steuer wie auch die Umverteilungsmacht des Steuerstaates zu schleichenden (Isensee 1977) oder gar offenen Verfassungstransformationen einladen (Abendroth 1968 [1954]). Doch die gegenwärtige Doppelkrise offenbart die Notwendigkeit zu politologischer Steuer- und Staatsfinanzforschung in besonderem Maße. Bislang wurde die Nachfrage insbesondere durch die Staatstätigkeitsforschung mit diversen Schwerpunktheften bedient: Während in der moderne staat erörtert wurde, ob Demokratien sparen können (Zohlnhöfer 2012), wurde anderorts festgestellt, dass sich die ,öffentlichen Finanzen im Umbruch“ (Wagschal 2014) und die „Schuldenkrise im Wandel“ (Enderlein 2014) befänden. Nur am Rande des politologischen Mainstreams wurde der dort deutliche politikfeldspezifische Zuschnitt um staats- und demokratietheoretische sowie steuer- und verteilungspolitische Fragen erweitert. ${ }^{3}$ Dieser Erweiterung nimmt sich auch der vorliegende Schwerpunkt an, der die genannten Problemstellungen mit einem breit gefächerten Blick aus den Teildisziplinen der Politikwissenschaft untermauert und untersucht.

\section{3 Überblick über die Beiträge}

Dem vorgenannten Anspruch, verschiedene Teildisziplinen der Politikwissenschaft zu den Demokratieproblemen des Steuerstaates zu Wort kommen zu lassen, hätte in Deutschland allerdings lange Zeit nicht nachgekommen werden können. Zu abseitig schien der jungen Disziplin eine Auseinandersetzung mit den öffentlichen Finanzen in ihrer Gründungsphase, wie Marc Buggeln aus wirtschafts- und wissenschaftshistorischer Sicht im ersten Beitrag des Schwerpunktheftes nachzeichnet. Erst die neomarxistischen Diskussionen über den Staat im Spätkapitalismus in den 1970er-Jahren und die Etablierung der Vergleichenden Wohlfahrtsstaatsforschung in

\footnotetext{
3 Siehe dazu die Ausgaben „Sparen und Herrschen“ und „Mit Steuern steuern“ der PROKLA (2009; 2011) sowie jüngst „Von Steuern und Staaten“ des Mittelweg 36 (2018).
} 
den 1980er-Jahren legten Grundsteine für eine etwas facettenreichere politologische Forschungsprogrammatik zum Fiskal- und Steuerstaat.

Dass und warum die Steuerstaatsfrage gleichwohl niemals konzeptionell in die moderne Politikwissenschaft übernommen worden ist, erläutert der Beitrag von Sebastian Huhnholz. Nach dem Ersten Weltkrieg wurden in den historisch, ökonomisch und gesellschaftspolitisch reflektierten Sozialwissenschaften zwar verschiedene Staatsfinanzierungsmodelle als signifikante Indikatoren unterschiedlicher politischer Regime verstanden. Insbesondere für Demokratien ließe sich damit fiskalische Souveränität als ein für die freiheitliche Regimesouveränität unerlässliches Gut bestimmen. Die Evidenz einer, politischen“ Identität der Fiskalverfassung ist aber nach dem Zweiten Weltkrieg nicht in eine alsbald wohlfahrtsstaatliche Politologie überführt worden, die nun verstärkt auf die innerdemokratische Distribution öffentlicher Güter blickte. Huhnholz plädiert dafür, die Demokratie selbst stärker als fiskalisch zu schützendes öffentliches Gut zu erfassen, um gegenwärtigen Trends, die eine umfassende Autorität über das Staatsbudget untergraben, zukünftig systematischer demokratietheoretischer Kritik zu unterwerfen und das Thema Steuerstaat für die Politikwissenschaft zu reklamieren.

Dabei sind nicht alle Facetten des Steuerstaates, das zeigt Laura Seelkopf in ihrem Artikel, exklusive Domänen westlicher Demokratien. In einem systematischen Vergleich von 138 Ländern über die letzten 30 Jahre erweist sich der Eindruck, dass Demokratien die ,besseren", d.h. progressiveren Steuerstaaten sind, als korrekturbedürftig. Autokratien, wenngleich unter anderen Legitimations- und Wettbewerbsanforderungen stehend, nutzen durchaus progressive Besteuerungsmaßnahmen und haben lange Zeit auch hohe Steuereinnahmen verbuchen können. Obgleich diese Parallele zu entwickelten Demokratien seit dem Ende des Kalten Krieges abgenommen hat, wirft Seelkopfs Beitrag die grundsätzliche Frage auf, ob Repression und Repräsentation äquivalente Prinzipien der Steuerstaatlichkeit sein können. Eine Frage, die angesichts der oben angedeuteten politischen Transformationen in der OECD-Welt eine unerwartete Dringlichkeit entwickeln könnte.

Politischen Dringlichkeiten wenden sich dann auch die Beiträge von Daniel Mertens und Thomas Rixen zu. So greift Mertens die weiterhin akute Krisensituation der Europäischen Union und insbesondere der Eurozone auf und unterzieht die auf ihre Lösung zielenden Vorschläge für eine weitere Vertiefung hin zur Fiskalunion einer demokratiepolitischen Diskussion. Die fiskalische Integration in Europa, so zeigt der Beitrag, hat die nationalen Steuerstaaten vor wachsende Herausforderungen gestellt, und weitere Integrationsschritte in diesem souveränitätssensiblen Bereich sind angesichts ihrer (für einige Länder fatalen) Eingriffstiefe höchst prekär. Die Effizienzkriterien und Stabilisierungsfunktion einer Fiskalunion, die bislang die Debatte bestimmen, sind daher nicht ausreichend, um die steigenden Legitimitätsanforderungen hinreichend zu beantworten. Rixen plädiert in seinem Beitrag für eine internationale Lösung zur Wahrung der fiskalischen Selbstbestimmung. Vor dem Hintergrund des die Handlungsfreiheit demokratischer Steuerstaaten einschränkenden Steuerwettbewerbs zeichnet er die normativen Grundlagen und legitimatorischen Erfordernisse nach, die eine internationale Koordinierung der Steuerpolitik unter den Bedingungen der Globalisierung zu erfüllen hat. Der Artikel kommt zu dem Ergebnis, dass eine spezifische Institutionalisierung der Steuerpolitik auf globaler Ebene in 
Form einer International Tax Organisation für die Staaten autonomie- und demokratieschonend sowie ökonomisch vorteilhaft sein kann. Er plädiert für die Aufnahme eines Reformdiskurses trotz aller gegenwärtigen Widrigkeiten.

Der Frage nach den normativen Grundlagen nationaler Steuerpolitik in einer internationalisierten Wirtschaft geht abschließend Gabriel Wollner nach. Aus dem Blickwinkel der analytischen Philosophie prüft er die Prämissen und Anforderungen, die fiskalische Interventionen und Selbstbestimmung rechtfertigen. Die Globalisierung interpretiert Wollner als Bedrohung für die institutionelle Form des demokratischen Steuerstaates, der nur mit einer ambitionierten Gegenstrategie zur Demokratisierung der Wirtschaft insgesamt beizukommen sei. In diesem Sinne ist der demokratische Steuerstaat eine historisch spezifische Erscheinung des nationalstaatlich gerahmten Kapitalismus, dessen Zukunft ungewiss und umstritten ist. Für eine normativ bewusste, theoretisch gehaltvolle und empirisch informierte Politikwissenschaft Aufruf genug, das Fiskalische nicht mehr aus den Augen zu verlieren.

Danksagung Unser Dank gilt allen beteiligten Autorinnen und Autoren, Lea Elsässer, Armin Schäfer und zwei anonymen Gutachterinnen und Gutachtern für hilfreiche Kommentare, sowie der PVS-Redaktion für die Zusammenarbeit. Ein gemeinsamer Workshop konnte im September 2016 dank der finanziellen Unterstützung der Deutschen Forschungsgemeinschaft aus Mitteln der Exzellenzinitiative an der LMU München durchgeführt werden.

\section{Literatur}

Abendroth, Wolfgang. 1968. Zum Begriff des demokratischen und sozialen Rechtsstaates im Grundgesetz der Bundesrepublik Deutschland. In Rechtsstaatlichkeit und Sozialstaatlichkeit, Hrsg. Ernst Forsthoff, 114-144. Darmstadt: Wiss. Buchgesellschaft. Orig. 1954.

Atkinson, Anthony B. 2015. Inequality: what can be done? Cambridge: Harvard University Press.

Biebricher, Thomas, und Frieder Vogelmann. 2014. Die Zukunft Europas zwischen Demokratie und Kapitalismus. Politische Vierteljahresschrift 55:1-17.

Bieling, Hans-Jürgen. 2015. Einleitung. In Steuerpolitik. Analysen, Konzeptionen, Herausforderungen, Hrsg. Hans-Jürgen Bieling, 5-7. Schwalbach: Wochenschau.

Brennan, Geoffrey, und James M. Buchanan. 1980. The power to tax. Analytical foundations of a fiscal constitution. Cambridge: Cambridge University Press.

Buchanan, James M., und Gordon Tullock. 1962. The calculus of consent. Logical foundations of constitutional democracy. Ann Arbor: University of Michigan Press.

Buggeln, Marc. 2012. Steuern nach dem Boom. Die Öffentlichen Finanzen in den westlichen Industrienationen und Ihre gesellschaftliche Verteilungswirkung. Archiv für Sozialgeschichte 52:46-89.

Buggeln, Marc. 2018. Ein bisschen Theorie und wenig Empirie: Die öffentlichen Finanzen als Thema der bundesrepublikanischen Politikwissenschaft (1949-1989). Politische Vierteljahresschrift 59(1). https://doi.org/10.1007/s11615-018-0052-5.

Downs, Anthony. 1960. Why the government budget is too small in a democracy. World Politics 12:541-563.

Drüen, Klaus-Dieter. 2015. Staatliche und private Finanzierung öffentlicher Aufgaben. In Subsidiarität in der Finanzverfassung, Hrsg. Hanno Kube, Ekkehart Reimer, 83-116. Heidelberg: Inst. für Finanzund Steuerrecht.

Enderlein, Henrik. 2013. Das erste Opfer der Krise ist die Demokratie. Wirtschaftspolitik und ihre Legitimation in der Finanzmarktkrise 2008-2013. Politische Vierteljahresschrift 54:714-739.

Enderlein, Henrik (Hrsg.). 2014. Schuldenkrise im Wandel. Politische Vierteljahresschrift 55.

Gawel, Erik. 2000. Das Steuerstaatsgebot des Grundgesetzes. Ein finanzwissenschaftlicher Beitrag zu seiner Inhaltsbestimmung. Der Staat 39:209-225.

Genschel, Phillip. 2003. Globalisierung als Problem, als Lösung und als Staffage. In Die neuen Internationalen Beziehungen - Forschungsstand und Perspektiven in Deutschland, Hrsg. Gunther Hellmann, Klaus Dieter Wolf, und Michael Zürn, 429-464. Baden-Baden: Nomos. 
Genschel, Phillip, und Peter Schwarz. 2013. Tax competition and fiscal democracy. In Politics in the age of austerity, Hrsg. Armin Schäfer, Wolfgang Streeck, 59-83. Cambridge: Polity.

Genschel, Phillip, und Susanne Uhl. 2006. Der Steuerstaat und die Globalisierung. In Transformationen des Staates?, Hrsg. Stephan Leibfried, Michael Zürn, 92-119. Frankfurt am Main: Suhrkamp.

Gilens, Martin. 2012. Affluence and influence: economic inequality and political power in america. New York: Princeton University Press.

Goldscheid, Rudolf. 1976. Staat, öffentlicher Haushalt und Gesellschaft. In Die Finanzkrise des Steuerstaats. Beiträge zur politischen Ökonomie der Staatsfinanzen, Hrsg. Rudolf Hickel, 253-316. Frankfurt am Main: Suhrkamp. Orig. 1926.

Gould, Andrew C., und Peter J. Baker. 2002. Democracy and taxation. Annual Review of Political Science 5:87-110.

Grauhan, Rolf Richard, und Rudolf Hickel (Hrsg.). 1978. Krise des Steuerstaates? Widersprüche, Perspektiven, Ausweichstrategien. Opladen: Westdeutscher Verlag.

Habermas, Jürgen. 1973. Legitimationsprobleme im Spätkapitalismus. Frankfurt am Main: Suhrkamp.

Habermas, Jürgen. 1998. Die postnationale Konstellation. Politische Essays. Frankfurt am Main: Suhrkamp.

Habermas, Jürgen. 2013. Demokratie oder Kapitalismus? Vom Elend der nationalstaatlichen Fragmentierung in einer kapitalistisch integrierten Weltgesellschaft. Blätter für deutsche und internationale Politik 10:59-70.

Haffert, Lukas. 2016. Die schwarze Null. Über die Schattenseiten ausgeglichener Haushalte. Berlin: Suhrkamp.

Hassel, Anke, und Susanne Lütz. 2010. Durch die Krise aus der Krise? Die neue Stärke des Staates. der moderne staat 3:251-271.

Herbert, Matis, und Dieter Stiefel. 1997. Krise des Steuerstaats - Steuerstaat in der Krise? Plädoyer für einen Funktionswandel des modernen Steuerstaats. Wien: Ueberreuter.

Hettich, Walter, und Stanley L. Winer. 1999. Democratic choice and taxation. A theoretical and empirical analysis. Cambridge: Cambridge University Press.

Homburg, Stefan. 2000. Allgemeine Steuerlehre. München: Vahlen.

Höpner, Martin, und Armin Schäfer. 2012. Embeddedness and regional integration: waiting for Polanyi in a Hayekian setting. International Organization 66:429-455.

Huhnholz, Sebastian. 2015. Refeudalisierung des Steuerstaates? Vorüberlegungen zu einer politischen Theorie der Steuerdemokratie. In Verfassung und Verteilung. Beiträge zu einer Grundfrage des Verfassungsverständnisses, Hrsg. Sigrid Boysen, Anna-Bettina Kaiser, und Florian Meinel, 175-216. Tübingen: Mohr Siebeck.

Huhnholz, Sebastian. 2017. Die Steuer des Steuerstaates. In Politik und Recht, Hrsg. Roland Lhotta, 454-472. Baden-Baden: Nomos.

Huhnholz, Sebastian. 2018a. Demokratie als öffentliches Gut? Die Finanzierung souveräner Freiheit im Steuerstaat. Politische Vierteljahresschrift 59(1). https://doi.org/10.1007/s11615-017-0042-z.

Huhnholz, Sebastian (Hrsg.). 2018b. Fiskus - Verfassung - Freiheit. Politisches Denken der öffentlichen Finanzen von Hobbes bis heute. Baden-Baden: Nomos.

Isensee, Josef. 1977. Steuerstaat als Staatsform. In Hamburg, Deutschland, Europa. Beiträge zum deutschen und europäischen Verfassungs-, Verwaltungs- und Wirtschaftsrecht, Hrsg. Rolf Stödter, Werner Thieme, 409-436. Tübingen: Mohr.

Kirchhof, Paul. 1996. Die verfassungsrechtliche Rechtfertigung der Steuern. In Steuern im Verfassungsstaat, Hrsg. Paul Kirchhof, Dieter Birk, und Moris Lehner, 27-53. München: C.H. Beck.

Knill, Christoph. 2013. Der Gebührenstaat: Latente und manifeste Gebührenpolitik im Spannungsfeld rechtlicher und wirtschaftlicher Rahmenbedingungen. der moderne staat 6:127-129.

Levi, Margaret. 1988. Of rule and revenue. Berkeley: University of California Press.

Mann, Fritz Karl. 1978. Steuerpolitische Ideale. Vergleichende Studien zur Geschichte der ökonomischen und politischen Ideen und ihres Wirkens in der öffentlichen Meinung 1600-1935. Darmstadt: WBG. Orig. 1937.

Mertens, Daniel. 2018. Demokratie oder Fiskalunion? Herausforderungen und Strukturprobleme der fiskalischen Integration in Europa. Politische Vierteljahresschrift 59(1). https://doi.org/10.1007/s11615018-0057-0.

Mittelweg 36. 2018. Von Steuern und Staaten. Mittelweg 3627.

Offe, Claus. 1972. Strukturprobleme des kapitalistischen Staates. Aufsätze zur Politischen Soziologie. Frankfurt am Main: Suhrkamp.

Offe, Claus. 2016. Europa in der Falle. Berlin: Suhrkamp.

Piketty, Thomas. 2014. Das Kapital im 21. Jahrhundert. München: C.H. Beck. 
Preuß, Ulrich K. 1981. Rechtsstaat - Steuerstaat - Sozialstaat. Eine Problemskizze. In Ordnungsmacht?: über das Verhältnis von Legalität, Konsens und Herrschaft, Hrsg. Dieter Deiseroth, Friedhelm Hase, und Karl-Heinz Ladeur, 46-68. Frankfurt am Main: Europäische Verlagsanstalt.

Priddat, Birger P. 2009. Politische Ökonomie. Neue Schnittstellendynamik zwischen Wirtschaft, Gesellschaft und Politik. Wiesbaden: Springer VS.

PROKLA 154. 2009. Mit Steuern steuern. PROKLA. Zeitschrift für kritische Sozialwissenschaft 39.

PROKLA 163. 2011. Sparen und Herrschen. PROKLA. Zeitschrift für kritische Sozialwissenschaft 41.

Reimer, Ekkehart. 2013. Die sieben Stufen der Steuerrechtfertigung. In Demokratie und Wirtschaft. Eine interdisziplinäre Herausforderung, Hrsg. Boris Gehlen, Frank Schorkopf, 113-141. Tübingen: Mohr Siebeck.

Rittberger, Berthold, und Frank Schimmelfennig. 2015. Kontinuität und Divergenz. Die Eurokrise und die Entwicklung europäischer Integration in der Europaforschung. Politische Vierteljahresschrift 56:389-405.

Rixen, Thomas. 2011. Tax Competition and Inequality: The Case for Global Tax Governance. Global Governance: A Review of Multilateralism and International Organizations 17(4): 447-467.

Rixen, Thomas. 2018. Globalisierung und fiskalische Demokratie. Politische Vierteljahresschrift 59(1). https://doi.org/10.1007/s11615-018-0060-5.

Ross, Michael L. 2004. Does taxation lead to representation. British Journal of Political Science 34:229-249.

Sacksofsky, Ute, und Joachim Wieland (Hrsg.). 2000. Vom Steuerstaat zum Gebührenstaat. Baden-Baden: Nomos.

Schäfer, Armin. 2013. Liberalization, inequality, and democracy's discontent. In Politics in the age of austerity, Hrsg. Armin Schäfer, Wolfgang Streeck, 169-195. Cambridge: Polity Press.

Scharpf, Fritz W. 1999. Regieren in Europa: Effektiv und Demokratisch? Frankfurt am Main: Campus.

Schumpeter, Joseph A. 1918. Die Krise des Steuerstaates. Graz: Leuschner \& Lubensky.

Seelkopf, Laura. 2018. Sind Demokratien die besseren Steuerstaaten? Ein globaler Vergleich, 1980-2010. Politische Vierteljahresschrift 59(1). https://doi.org/10.1007/s11615-018-0059-y.

Steinmo, Sven. 1993. Taxation and democracy. Swedish, British and American approaches to financing the modern state. New Haven, London: Yale University Press.

Stiglitz, Joseph E. 2008. The price of inequality. New York: Norton \& Company.

Strange, Susan. 1996. The retreat of the state: the diffusion of power in the world economy. Cambridge: Cambridge University Press.

Streeck, Wolfgang. 1998. Einleitung: Internationale Wirtschaft, Nationale Demokratie? In Internationale Wirtschaft, nationale Demokratie: Herausforderungen für die Demokratietheorie, Hrsg. Wolfgang Streeck, 11-58. Frankfurt am Main: Campus.

Streeck, Wolfgang. 2013. Gekaufte Zeit: Die vertagte Krise des demokratischen Kapitalismus. Berlin: Suhrkamp.

Streeck, Wolfgang, und Daniel Mertens. 2010. Politik im Defizit. Austerität als fiskalpolitisches Regime. der moderne staat 3:7-29.

Streeck, Wolfgang, und Daniel Mertens. 2013. Public finance and the decline of state capacity in democratic capitalism. In Politics in the age of austerity, Hrsg. Armin Schäfer, Wolfgang Streeck, 26-58. Cambridge: Polity.

Wagschal, Uwe. 2003. Politische Ökonomie der Besteuerung. In Politische Ökonomie. Demokratie und wirtschaftliche Leistungsfähigkeit, Hrsg. Herbert Obinger, Uwe Wagschal, und Bernhard Kittel, 259-288. Opladen: Leske + Budrich.

Wagschal, Uwe (Hrsg.). 2014. Die öffentlichen Finanzen im Umbruch. Zeitschrift für Staats- und Europawissenschaften 12:137-152.

Weber, Max. 2014. Ordnung der Finanzierung politischer Verbände. In Wirtschaft und Gesellschaft. Soziologie. Unvollendet 1919-1920 Studienausgabe der Max-Weber-Gesamtausgabe, Bd. I, 143-146. Tübingen: J.B.C. Mohr. Orig. 1919-1920.

Wollner, Gabriel. 2018. Globale Besteuerung und Demokratische Legitimität: Moralische Herausforderungen an den demokratischen Steuerstaat. Politische Vierteljahresschrift 59(1). https://doi.org/10.1007/ s11615-018-0056-1.

Zohlnhöfer, Reimut. 2012. Die Schwierigkeiten der Demokratie mit dem Sparen. Eine Einleitung. der moderne staat 5:263-267.

Zürn, Michael. 1998. Regieren Jenseits des Nationalstaates. Globalisierung und Denationalisierung als Chance. Frankfurt am Main: Suhrkamp. 